\title{
La misura della qualità di vita e dell'utilità in farmacoeconomia: strumenti e loro applicazioni in una rassegna di letteratura

Ya-Ting Chan*

\begin{abstract}
In a climate of economic scarcity, decisions about the allocation of healthcare resources need to be made explicit. An economic evaluation is able to identify and quantify the relationship between an input of resources and an output, hence to appreciate the efficiency of the project. One of the recent methodological approaches that has been used increasingly in health economic evaluations is the 'cost-utility analysis' (CUA). CUA considers benefits in utility terms and uses a unique index to assess them. We investigated the generic measurements of health related quality of life in the economic evaluation. Different instruments for evaluating quality of life and utility were categorized and briefly introduced. Literatures published in the journal "PharmacoEconomics" in 1997-2001 regarding quality of life and utility were reviewed. We were especially interested in the application of health profile SF-36 and utility measure EQ-5D. Selected articles were further analyzed and some issues concerning the methodologies of quality of life and utility are discussed.
\end{abstract}

Farmeconomia e percorsi terapeutici 2002; 3 (4): 241-252

\section{SOMMARIO}

Abbiamo esaminato gli strumenti generici di misura della qualità di vita correlata alla salute utilizzati nelle valutazioni economiche. I differenti strumenti usati per misurare la qualità della vita e l'utilità sono stati suddivisi in categorie e brevemente introdotti. Sono state prese in rassegna le pubblicazioni relative alla qualità di vita comparse sulla rivista "PharmacoEconomics" nel periodo 1997-2001. Il nostro interesse era rivolto principalmente alle applicazioni del Profilo di salute SF-36 e della misura d'utilità EQ-5D. Sono stati inoltre ulteriormente analizzati alcuni articoli selezionati e discussi alcuni problemi metodologici relativi alla misura della qualità di vita e dell'utilità.

\section{QUALITÀ DI VITAEUTILITÀ}

In un ambito di scarsità, le decisioni riguardanti l'allocazione delle risorse sanitarie devono essere rese esplicite (1). Una valutazione economica è in grado di identificare e quantifi- care la relazione esistente tra l'input di risorse (i costi) e l'output (i benefici), dunque di valutare l'efficienza di un progetto (2). Tra i recenti approcci metodologici, l'analisi costo-utilità (CUA) è stata utilizzata con frequenza crescente nelle valutazioni economiche in ambito sanitario. La CUA misura i benefici in termini di utilità e utilizza un indicatore sintetico per valutarli. I rapporti di costo/efficacia misurati con la CUA sono pertanto sempre paragonabili tra loro.

La misura dello stato di salute è una tappa cruciale nelle valutazioni economiche. La definizione di salute proposta mezzo secolo fa (1947) dall'OMS: "uno stato di benessere fisico, mentale e sociale completo, e non mera assenza di malattia", ha determinato una rivoluzione nelle valutazioni della salute. La qualità di vita correlata alla salute è il risultato concettuale di questo processo di revisione, radicato nella convinzione che le cure sanitarie non debbano solamente prolungare il corso della vita, ma anche migliorarne la qualità: "non aggiungete solo anni alla vita, ma anche vita agli anni" è lo slogan che riassume questo atteggiamento. Tale concetto considera lo stato di salute
* Master of International Health Care Management, Economics and Policy (MIHMEP)

SDA Bocconi 


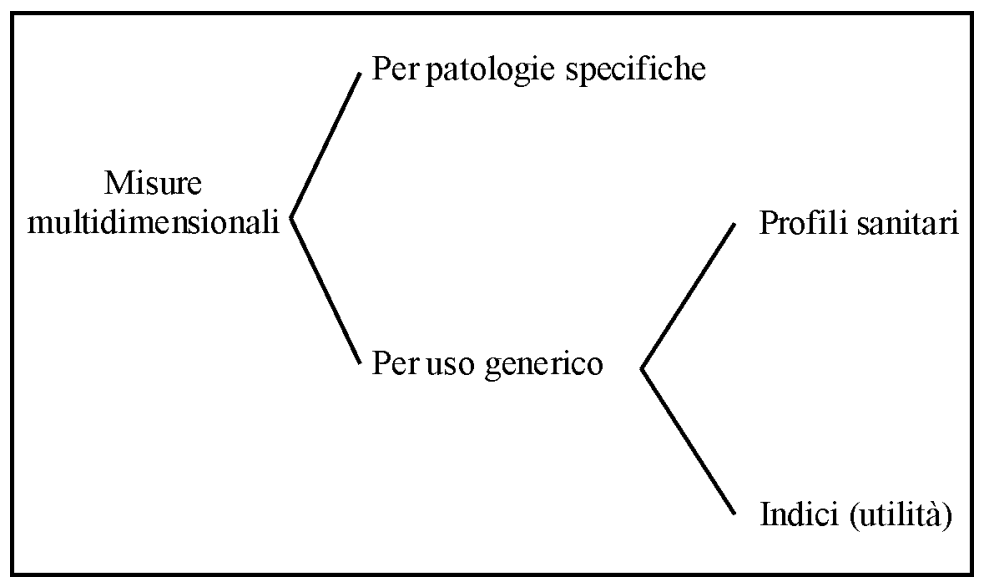

Figura 1

nella sua accezione più ampia e completa: la qualità di vita ("correlata alla salute", in quanto le variabili sanitarie sono le sole considerate per la valutazione di tale qualità).
Data la complessità del parametro qualità di vita, le misure multidimensionali, che racchiudono componenti fisiche, mentali e sociali in un unico strumento, sono le più adeguate per "catturarlo". In questa rassegna trascureremo gli strumenti relativi a patologie specifiche. D'altro canto, gli strumenti adatti ad un utilizzo generale possono essere suddivisi in due grosse sottoclassi: i profili e gli indicatori sintetici.

I profili riassumono le informazioni sui livelli di salute nelle dimensioni considerate (ad esempio fisica, mentale, sociale) ma non in maniera trasversale ad esse, senza cioè cercare di ottenere un indice sintetico complessivo dello stato di salute. Si tratta sostanzialmente di misure descrittive e sebbene con alcune di esse sia possibile costruire un singolo punteggio complessivo, ciò non è il loro obiettivo princi-

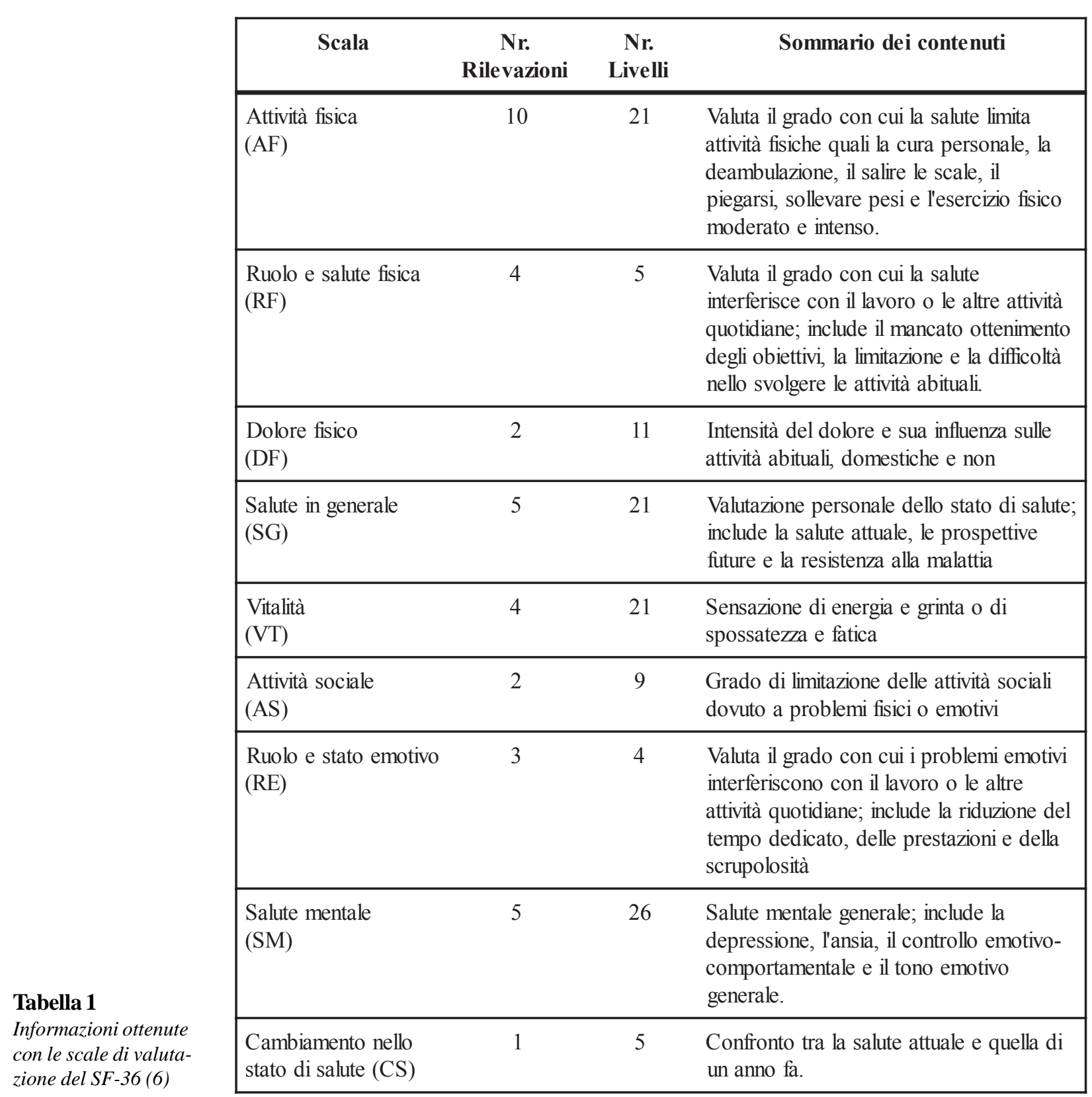


pale. Questi strumenti generici possono essere applicati a una gran varietà di popolazioni, essi sono adeguati per un confronto dell'impatto relativo di differenti programmi sanitari.

Gli indicatori sintetici appaiono essere gli strumenti adeguati per la valutazione dell'utilità, che è un punteggio numerico che misura in maniera sintetica la qualità di vita, ma non è la qualità di vita intesa in senso stretto. L'utilità è un concetto di pertinenza dell'economista, in quanto misura la soddisfazione causata da (o la preferenza espressa per) una determinata scelta. È stata adottata nell' ambito delle valutazioni come un tipo particolare di misura della soddisfazione fornita dalla qualità di vita conseguente a un determinato stato di salute. Gli elementi chiave delle misure di utilità risiedono nell'inclusione di misure di preferenza e nel relazionare gli stati di salute alla morte. Ciò permette il loro utilizzo nelle analisi costo/utilità che combinano durata e qualità della vita.

\section{PROFILISANITARI: MISURE DIQUALITÀ DI VITA}

I profili sanitari forniscono una serie di punteggi, riferiti a singole dimensioni dell'HRQOL (Health-Related Quality Of Life). Uno dei vantaggi dei profili sanitari consiste nella produzione di punteggi di esito plurimi, che possono consentire a clinici e ricercatori una valutazione differenziata degli effetti di una patologia, o del suo trattamento, sui vari domini HRQOL. In alcuni casi, inoltre, sono stati derivati dei punteggi riassuntivi, calcolati sulla media dei punteggi ottenuti nei vari sottogruppi della qualità di vita considerati dal profilo (3).

\section{Medical Outcomes Study 36-Item Short- Form Questionnaire (MOS-SF-36)}

Il questionario per la rilevazione dello stato di salute MOS SF-36, uno tra gli strumenti di valutazione dell'HR-QOL più diffusi, è un questionario generico che indaga due dei principali aspetti della salute (fisico e mentale) attraverso 36 item, suddivise in otto dimensioni: attività fisica $(A F)$, ruolo e salute fisica $(R F)$, dolore fisico (DF), salute in generale (SG), vitalità (VT), attività sociali (AS), ruolo e stato emotivo (RE) e salute mentale (SM). È inoltre disponibile una misura di autovalutazione del cambiamento dello stato di salute (4).

Sono state derivate scale riassuntive delle componenti fisiche (PCS) e mentali (MCS) del questionario. Sono inoltre in corso alcuni tentativi di convertire i risultati del SF-36 in un'unica misura di utilità (5).

\section{Nottingham Health Profile (NHP)}

L'NHPè stato sviluppato in Gran Bretagna e consiste di due parti. Oggi viene utilizzata solo la prima parte che valuta la percezione soggettiva della salute, richiedendo all' esaminato di rispondere "si" o "no" a 38 affermazioni, suddivise in sei sezioni (Tabella 2). Il punteggio viene normalmente calcolato separatamente per le varie sezioni, dopo ponderazione dei singoli elementi, e varia da 0 (nessun problema) a 100 (tutti i problemi segnalati); punteggi bassi indicano una buona funzionalità.

\section{Sickness Impact Profile (SIP)}

Il SIP contiene 136 rilevazioni, suddivise nelle 12 categorie elencate in tabella 2 . Fatta eccezione per 5 categorie indipendenti, le altre possono essere ulteriormente aggregate in due dimensioni principali: fisica e psicosociale.

Ogni rilevazione è composta di una descrizione di un'attività della vita quotidiana, espressa in prima persona e con il presente indicativo. Il punteggio attribuito alle singole attività viene ponderato in base al grado di disfunzione che rappresentano. Tale ponderazione è frutto del lavoro di una commissione composta da oltre 100 esperti, che hanno attribuito un punteggio ad ogni attività utilizzando una scala di 11 livelli a intervallo fisso, da "disfunzione minima" a "disfunzione grave". I pazienti devono segnare solamente le affermazioni che descrivono lo stato della loro salute nel periodo esaminato. Il punteggio del SIP si basa sul prodotto del numero di attività segnate con il loro peso relativo: tale valore viene diviso per il massimo punteggio

\begin{tabular}{|ll|}
\hline \multicolumn{1}{|c|}{ NHP } & \multicolumn{1}{c|}{ SIP } \\
\hline Livello energetico & Dimensione fisica \\
Reazioni emotive & Deambulazione \\
Mobilità fisica & Mobilità \\
Dolore & Cura personale e motilità \\
Isolamento sociale & Dimensione psicosociale \\
Sonno & Comunicazione \\
& Raziocinio \\
& Emotività \\
& Relazioni sociali \\
& Categorie indipendenti \\
& Sonno e riposo \\
& Alimentazione \\
& Lavoro \\
& Gestione domestica \\
& Attività ricreative e passatempi \\
\hline
\end{tabular}

Tabella 2

Aree tematiche di NHP e SIP 
possibile e moltiplicato per 100. Il punteggio, pertanto, rappresenta una percentuale della massima disfunzione prevista: 0 rappresenta la situazione ideale e 100 la mancanza totale di funzionalità. I punteggi vengono calcolati separatamente per le varie categorie e dimensioni.

\section{Il questionario WHO QOL}

Il questionario sulla qualità di vita dell'Organizzazione Mondiale della Sanità (WHOQOL100) è uno strumento HR-QOL generico, di concezione multidimensionale, composto da 100 elementi. Le domande indagano la percezione e la valutazione soggettiva della qualità di vita generale e della salute del paziente, oltre a esplorare 6 ampi domini della sua vita. Ogni

\begin{tabular}{|l|}
\hline FISICO \\
\hline Dolore e fastidio fisico \\
Energia e fatica \\
Sonno e riposo \\
\hline PSICOLOGICO \\
\hline Sensazioni positive \\
Pensiero, apprendimento, memoria e concentrazione \\
Autostima \\
Immagine e aspetto fisico \\
Sensazioni negative \\
\hline LIVELLO DI INDIPENDENZA \\
\hline Mobilità \\
Attività della vita di tutti i giorni \\
Dipendenza da farmaci/trattamenti \\
Capacità lavorativa \\
\hline RELAZIONI SOCIALI \\
\hline Relazioni personali \\
Assistenza sociale \\
Attività sessuale \\
\hline AMBIENTE \\
\hline Sicurezza \\
Ambiente domestico \\
Risorse finanziarie \\
Assistenza sociosanitaria: disponibilità e qualità \\
Possibilità di acquisire nuove informazioni e abilità \\
\hline SPIRITUALitualità/religione/convinzioni personali \\
\hline
\end{tabular}

Tabella 3

WHO QOL: domini e sfaccettature dominio comprende una serie di aspetti (sottodomini) della qualità di vita, che ne riassumono le caratteristiche salienti. La struttura del WHOQOL è rappresentata in tabella 3.

\section{MISUREDIUTILITÀ (BASATESULLEPREFERENZE)}

Nella valutazione della HR-QOL, le misure basate sulla preferenza forniscono un unico numero, che si inserisce in un continuum compreso tra la salute perfetta (1) e la morte (0). Tale singolo valore numerico rappresenta lo stato di salute soggettivamente percepito dall' esaminato in un determinato momento ed esprime la preferenza o l'utilità per quel dato stato di salute. La differenza tra valori di preferenza e utilità viene discussa dettagliatamente altrove (8).

Un metodo comune per incorporare le preferenze nelle valutazioni HR-QOL consiste nell'utilizzo di sistemi di classificazione dello stato di salute ad attributi multipli. Tali misure forniscono una descrizione degli stati di salute; per calcolare il punteggio si applica una funzione di preferenza riferita agli stati di salute indagati. Le funzioni di preferenza sono state stimate mediante studi di popolazione condotti con l'utilizzo di tecniche diverse, quali lo standard-gamble, il time trade-off e/o le scale ad analogia visiva.

\section{Tecniche di valutazione di uno stato di salute}

Queste tecniche richiedono al paziente, o a chi per lui, di dare un giudizio su un certo stato di salute. Per quanto semplici ed efficienti, queste misure ad attributo singolo forniscono informazioni su di un unico stato di salute.

\section{Visual Analogue Scale (VAS) o Rating Scale (RS)}

Le scale analogiche visive (VAS) sono semplicemente delle linee, normalmente comprese tra due estremi ben definiti, sulle quali i pazienti possono indicare la loro preferenza. Le linee possono variare in lunghezza e orientamento e possono o meno essere suddivise in intervalli marcati con valori numerici. Il compito richiesto è di giudicare la desiderabilità relativa di vari stati di salute, assegnando loro un valore numerico su di una scala analogica compresa tra 0 (morte) e 100 (stato di salute perfetto o massima salute desiderabile).

\section{Magnitude Estimation (ME)}

L'ME richiede ai pazienti di giudicare il rapporto di indesiderabilità tra coppie di stati di 
salute; ad esempio il paziente può riferire che lo stato A è x volte peggiore dello stato $\mathrm{B}$. L'indesiderabilità (o l'inutilità) dello stato $\mathrm{A}$, pertanto, è $\mathrm{x}$ volte maggiore di quella dello stato B. Con una serie di tali domande si possono rapportare tra loro tutti gli stati di salute sulla base della scala di indesiderabilità.

\section{Standard Gamble (SG)}

L'SG richiede al soggetto di operare una scelta tra due esiti alternativi, di cui uno incerto. Viene loro richiesto di stabilire quanto rischio di morte, o di altro esito peggiore di quello valutato, essi siano disposti ad accettare per evitare la certezza dello stato di salute valutato. Da molti considerato l'unico metodo di misura dell'utilità in senso stretto, l'SG si basa direttamente sugli assiomi della teoria originale dell'utilità attesa.

\section{Time Trade-Off (TTO)}

La tecnica TTO richiede al soggetto di esprimere la sua preferenza tra due alternative, entrambe caratterizzate da una determinata prospettiva temporale; ad esempio $\mathrm{x}$ anni in piena salute e t anni nello stato di salute da valutare. Al paziente viene richiesto di prendere in considerazione una riduzione della durata della vita in cambio di un miglioramento dello stato di salute. La valutazione dello stato di salute corrisponde alla frazione di anno di vita in salute equivalente ad un anno di vita nello stato di salute che si vuole valutare, ossia $\mathrm{x} / \mathrm{t}$.

\section{Misure ad attributi multipli EuroQol (EQ-5D)}

Lo strumento EuroQol (EQ-5D) è stato sviluppato congiuntamente da un gruppo di ricercatori attivi in Europa con lo scopo di costruire uno strumento semplice che fornisse un punteggio sintetico, indicativo della preferenza per un determinato stato di salute (11).

L'EQ-5D consta di 2 parti. La prima è un sistema descrittivo che definisce la HR-QOL mediante 5 dimensioni:

- Mobilità

- Cura personale

- Attività abituali

- Dolore/fastidio

- Ansia/depressione

Ogni dimensione prevede 3 livelli di gravità: nessun problema $=1$, problemi di entità moderata $=2$ e problemi di gravità estrema $=3$.

Uno stato di salute è definito dalla combinazione delle 5 dimensioni, ognuna con il livel- lo di gravità assegnato. Così lo stato di salute 11122, ad esempio, rappresenta uno stato in cui non sussistono problemi di mobilità, cura personale o nelle attività abituali, ma sono presenti discreti problemi nelle dimensioni dolore o fastidio fisico e ansia o depressione. Il sistema EQ-5D genera 243 possibili stati di salute, a cui vanno aggiunti l'assenza di coscienza e la morte. Ogni singolo stato di salute può essere tradotto in un punteggio mediante l'utilizzo di valori di preferenza (pesi) predeterminati, generati da terzi, ad esempio intervistando la popolazione generale.

La seconda parte dell'EQ-5D consiste in una VAS di $20 \mathrm{~cm}$ a intervalli premarcati, dove lo 0 all'inizio della linea corrisponde al peggior stato di salute immaginabile e l'altro estremo, segnato con 100, rappresenta il miglior stato di salute immaginabile. I soggetti utilizzano la VAS per valutare il proprio stato di salute nel giorno di somministrazione del test.

Evidenze sperimentali (36) hanno dimostrato che la correlazione tra i punteggi ottenuti con l'EuroQol-SC (self-classifier, prima parte) e con l'EuroQol-VAS (seconda parte) non è particolarmente forte (coefficiente di correlazione di Pearson =0,54). I due strumenti di misura dell'utilità, pertanto, riflettono giudizi in qualche misura differenti e non sembrano essere intercambiabili.

\section{Health Utility Index (HUI)}

L'HUI si compone di due parti: (i) un sistema di classificazione dello stato di salute ad attributi multipli per la descrizione dello stato di salute e (ii) una funzione di utilità ad attributi multipli che viene utilizzata per calcolare il valore dello stato di salute misurato. L'HUI1 considerava 4 attributi e forniva una formula per il calcolo dell'utilità. La seconda versione, l'HUI2, consiste di 7 attributi e di formule per il calcolo delle utilità e dei valori di preferenza. La versione più recente e potenzialmente più utile, l'HUI3, prende in considerazione 8 attributi (Tab.4). Recentemente è stata sviluppata una funzione di utilità moltiplicativa ad attributi multipli per quest'ultima versione. Essa si basa sui risultati di una VAS e di misure di standard gamble ottenuti da un campione randomizzato della popolazione generale di Hamilton, Ontario, Canada. L'HUI3 descrive 972000 differenti stati di salute.

\section{Quality of Well-Being (QWB)}

La scala QWB è stata uno dei primi strumenti introdotti nel campo dei sistemi di misura della qualità di vita o dello stato di salute. 
Essa valuta la HR-QOL utilizzando 3 scale che rappresentano aspetti della vita quotidiana collegati tra loro, ma differenti: mobilità (3 livelli), attività fisica (3 livelli) e attività sociale (5 livelli). Inoltre vengono valutati dei complessi sintomo/problema che rappresentano problemi di salute o sintomi clinici che possono interferire con la funzionalità o il benessere. La versione attuale del QWB comprende 26 complessi sintomo/problema, mentre la versione sperimentale per l'autocompilazione (QWB-SA scale) include 58 sintomi.

\section{SELEZIONEDELLALETTERATURA}

Per questa rassegna parziale sono stati considerati gli articoli originali pubblicati sulla rivista "PharmacoEconomics" tra il 1997 e il 2001 e indicizzati nel Subject Index sotto le voci CostUtility, Quality of life, Quality-of-life rating scales e Quality-adjusted-life-years. Sono state escluse le review e i lavori in cui venivano utilizzati solamente strumenti di misura specifici per patologia. Da questa ricerca sono emersi 29 articoli. $(6,7,10,12-37)$

Gli strumenti utilizzati sono stati molteplici.

15 articoli hanno adottato i profili sanitari per la misura della qualità di vita. Di questi 7 hanno impiegato il MOS SF-36, 3 hanno usato il NIP, mentre MOS SF-12, MOS-6A, SIP, FSQ e WHOQOL sono stati utilizzati una volta ciascuno.

Dieci lavori hanno invece adoperato strumenti di misura della preferenza multiattributo. EQ-5D è stato impiegato in 6 lavori, Health Utility Index (HUI) in 3 e Quality of Well-Being (QWB) in uno.

Nello studio di Griffin et al. (21) i valori di utilità non sono stati ricavati direttamente nel corso dello studio clinico, ma sono stati raccolti successivamente da due fonti: i) a un campione rappresentativo della popolazione generale, selezionato da un database britannico di pazienti di medicina generale, è stato richie-

\begin{tabular}{|lll|}
\hline \multicolumn{1}{|c|}{ EQ-5D } & \multicolumn{1}{c|}{ HUI 3 } & \multicolumn{1}{c|}{ QWB } \\
\hline Capacità di movimento & Visione & Mobilità \\
Cura personale & Udito & Attività fisica \\
Attività abituali & Linguaggio & Attività sociale \\
Dolore/fastidio & Deambulazione & Sintomi/problemi \\
Ansia/depressione & Destrezza & \\
& Emotività & \\
& Funzioni cognitive & \\
& Dolore & \\
\hline
\end{tabular}

Tabella 4

Aree tematiche delle misure basate sulla preferenza sto di compilare i questionari EQ-5D in maniera retrospettiva; ii) per avere un controllo sui valori generati dai pazienti, un panel di MMG britannici ha utilizzato l'EQ-5D per classificare lo stato di salute di un ipotetico paziente ad alto rischio; questi valori non sono poi stati utilizzati nell'analisi.

Cinque articoli hanno misurato la qualità di vita utilizzando indicatori singoli, mentre altri tre hanno usato i risultati di studi precedenti.

Trellori e Messori, assumendo che la qualità di vita dei pazienti affetti da morbo di Chron possa essere stratificata in soli 5 livelli, hanno richiesto ad un gruppo di 10 esperti gastroenterologi italiani di attribuire un punteggio di qualità di vita per ciascuno di questi 5 livelli (Tab. 5).

\section{Utilizzo di MOS SF-36}

Le metodologie impiegate nei 7 articoli che hanno usato il MOS SF-36 per valutare la qualità di vita sono analizzate ed elencate in tabella 6, che riporta anche il luogo della raccolta dati e le aree cliniche esplorate. Quattro lavori hanno valutato l'impatto della patologia sulla qualità di vita dei pazienti, due hanno misurato gli esiti del trattamento in termini di qualità di vita e uno era studiato per verificare la robustezza dell'SF-36 nel valutare pazienti asintomatici HIV-positivi. Il tasso di risposta ai questionari è piuttosto vario ed è probabilmente correlato alla maniera di presentazione del test e alla presenza di incentivi. Per esempio, il tasso di risposta più alto osservato nei sondaggi condotti per posta si è avuto dove veniva offerta una ricompensa per la compilazione. Ciò potrebbe comportare un bias del campionamento. Quattro dei 7 studi hanno utilizzato i valori normali della popolazione generale come misura di controllo. In uno di questi studi, vista l'indisponibilità di valori normali di riferimento per la popolazione locale, sono stati utilizzati i valori di due paesi stranieri. Gli altri tre lavori non prevedevano l'utilizzo di un campione di controllo, bensì hanno confrontato $i$ risultati tra sottogruppi dello stesso studio o tra fasi diverse del trattamento.

\section{Utilizzo della misura di utilità (EQ-5D)}

Le metodologie impiegate nei 6 articoli che hanno usato l'EQ-5D per valutare la qualità di vita sono analizzate ed elencate in tabella 7 , che riporta anche il luogo della raccolta dati e le aree cliniche esplorate. Solo uno di essi ha esplorato gli effetti di una specifica malattia sull'utilità dei pazienti, mentre un altro ha valutato le conseguenze dell'esperienza di malattia sulla percezione personale dello stato di salute. Due di essi hanno valutato gli esiti, in 
Misura dello stato di salute/utilità

Lavoro

Ackerman SJ. et al.

Aoki FY. et al.

Arpinelli F. et al.

Badia X. et al.

Boyer P. et al.

Coyle D. et al.

Cremieux PY. et al.

Dahlöf C. et al.

Evans KW. et al.

Fagnani F. et al.

Griffin AD. et al.

Griffiths RI. et al.

Gudex CM. et al.

Hakkaart-van Roijen L et al.

Hatoum HT. et al.

Hummel S. et al.

Johnson JA. et al.

Mittmann N. et al.

O'Conor RM. et al.

Pariente PD. et al.

Pibernik-Okanovic $\mathrm{M}$ et al.

Rosner AJ. et al.

Shimbo T. et al.

Smith KJ. et al.

Torrance G. et al.

Trallori G. et al.

Trippoli S. et al.

Uyl-de Groot CA.et al.

Voruganti L. et al.
Profilo sanitario

(misure di qualità di vita)

\section{Basate sulla preferenza} (misure di utilità)

SG

MOS-6A

MOS SF- 36

EQ-5D

FSQ

VAS

VAS

MOS SF- 36

NHP

QWB

EQ-5D di 2 lavori precedenti

Lavoro precedente

EQ-5D

MOS SF-36

MOS SF-36

MOS SF-12

Lavoro precedente

EQ-5D

HUI-3

MOS SF-36

MOS SF-36

WHOQOL

$\begin{array}{cc} & \text { HUI-2 } \\ & \text { TTO } \\ & \text { Lavoro precedente } \\ \text { NHP } & \text { HUI } \\ & \text { Expert opinion } \\ \text { MOS SF-36 } & \text { EQ-5D } \\ \text { NHP } & \text { EQ-5D } \\ \text { SIP } & \text { ME, RS, SG, TTO }\end{array}$

Tabella 5

Utilizzo degli strumenti generici di misura della Health-Related Quality of life negli articoli pubblicatisu: "PharmacoEconomics" dal 1997 al 2001
EQ-5D: EuroQoL,

HUI: Health Utilities Index,

QWB: Quality of Well-Being,

MOS SF-36, SF-12, 6A: Medical Outcomes

Study,

NHP: Nottingham Health Profile,

SIP: Sickness Impact Profile,
FSQ: Functional Status Questionnaire, WHOQOL: WHO QOL Questionnaire, VAS: Visual Analogue Scale,

SG: Standard Gamble, TTO: Time trade-off,

RS: Rating Scale,

ME: Magnitude Estimation 
termini di utilità, di farmaci specifici. In un lavoro è stato condotto un sondaggio su di un ampio campione per stabilire valori di riferimento per la popolazione generale. In Gran Bretagna erano disponibili due serie di dati di popolazione, che sono stati utilizzati in uno studio.

\section{DISCUSSIONE}

Questo lavoro ha esaminato l'utilizzo degli strumenti generici di valutazione della qualità di vita correlata alla salute in 29 articoli originali pubblicati su "Pharmacoeconomics" tra il 1997 e il 2001. Abbiamo rivolto la nostra attenzione particolarmente sui lavori che hanno adottato MOS SF-36 e EQ-5D come strumenti di misura della qualità di vita e dell'utilità, in quanto costituiscono i metodi più utilizzati.

In sei delle sette ricerche (Tabella 6) che lo hanno usato, il MOS SF-36 è servito a valutare il burden of disease, ossia l'impatto delle malattie sulla qualità di vita. Queste ricerche hanno cercato di valutare i costi intangibili in cui sono incorsi i pazienti attraverso la misurazio- ne della qualità di vita. In un altro lavoro, Arpinelli et al. hanno valutato la validità clinica e l'accuratezza delle misure psicometriche prodotte dal MOS SF-36 su di un campione italiano di pazienti asintomatici HIV-positivi. I risultati dimostrano che il profilo sanitario MOS SF-36 è adatto per effettuare misure psicometriche in queste condizioni e i risultati prodotti ne dimostrano validità e robustezza. Hakkaart-van Rojen et al., Hatoum et al. e Trippoli et al. hanno osservato significative associazioni tra HR-QOL ridotta e vari domini del MOS SFR-36 in pazienti con specifiche patologie rispetto alla popolazione generale. Due di essi (Hakkaart-van Rojen e Hatoum) hanno anche riscontrato un maggior consumo di risorse sanitarie in questi pazienti.

Trippoli et al. (36) hanno deciso di applicare contemporaneamente due strumenti concettualmente differenti, MOS SF-36 ed EuroQOL, a 95 pazienti affetti da cancro polmonare non a piccole cellule (NSCLC) trattati in 15 ospedali italiani e hanno riscontrato una forte correlazione tra i risultati ottenuti con i due tipi di misura. In media, la somma dei punteggi delle dimensioni "fisiche" dell'SF-36 è ri-

\begin{tabular}{|c|c|c|c|c|c|c|}
\hline Lavoro & Luogo & Area clinica & Obiettivo & $\begin{array}{l}\text { Dimensione } \\
\text { del campione }\end{array}$ & Somminis trazione & $\begin{array}{l}\text { Gruppo di } \\
\text { controllo }\end{array}$ \\
\hline Pariente PD. et al. & Francia & Rinite Perenniale & $\begin{array}{l}\text { Esiti Qol e uso } \\
\text { di antistaminici }\end{array}$ & $\begin{array}{l}1367 \\
1097(80 \%) \\
\text { complete }\end{array}$ & self, per posta & (3 sottogruppi) \\
\hline Dahlöf C. et al. & $\begin{array}{l}\text { Australia, } \\
\text { Canada, } \\
\text { Germania, Italia, } \\
\text { Svezia }\end{array}$ & Emicrania & $\begin{array}{l}\text { HR-QOL del } \\
\text { Sumatriptan } \\
\text { sottocutaneo }\end{array}$ & $\begin{array}{l}749 \\
482(64 \%) \\
\text { complete }\end{array}$ & self, in clinica & $\begin{array}{l}\text { (tra } 4 \text { fasi di } \\
\text { trattamento) }\end{array}$ \\
\hline $\begin{array}{l}\text { Hakkaart-van } \\
\text { Roijen L et al. }\end{array}$ & Belgio & $\begin{array}{l}\text { GHD } \\
\text { ipopituitaria }\end{array}$ & $\begin{array}{l}\text { Burden of } \\
\text { disease }\end{array}$ & $\begin{array}{l}215 \\
138(75 \%) \\
\text { risposte, } 129 \\
\text { incluse }\end{array}$ & self, per posta & $\begin{array}{l}\text { Valori di } \\
\text { riferimentoUSA } \\
\text { e Olanda }\end{array}$ \\
\hline O'Conor RM. et al. & USA & $\begin{array}{l}\text { Urge } \\
\text { Incontinence }\end{array}$ & $\begin{array}{l}\text { Effetti della } \\
\text { patologia sulla } \\
\text { Qol }\end{array}$ & $495(83.8 \%)$ & $\begin{array}{l}\text { self, per posta } \\
\text { ricompensa di } \\
\text { US } \$ 20 \text { per } \\
\text { partecipazione }\end{array}$ & $\begin{array}{l}\text { Valori di } \\
\text { riferimentoUSA }\end{array}$ \\
\hline Hatoum HT. et al. & USA & Insonnia & $\begin{array}{l}\text { Effetti della } \\
\text { patologia sulla } \\
\text { Qol }\end{array}$ & $\begin{array}{l}7500 \\
1740(32 \%) \text { per } \\
\text { posta } 1707 \\
(85 \%) \text { in clinica }\end{array}$ & $\begin{array}{l}\text { self, } \\
5500 \text { per posta } \\
2000 \text { in clinica }\end{array}$ & $\begin{array}{l}\text { Tra assenza di } \\
\text { insonnia, } \\
\text { insonnia di primo } \\
\text { e insonnia di } \\
\text { secondo livello }\end{array}$ \\
\hline Arpinelli F. et al. & Italia & $\begin{array}{l}\mathrm{HIV} \\
\text { asintomatico }\end{array}$ & $\begin{array}{l}\text { Prestazione } \\
\text { psicometrica e } \\
\text { validità clinica }\end{array}$ & $\begin{array}{l}214 \\
201(94 \%) \\
\text { valutabili }\end{array}$ & self, in clinica & $\begin{array}{l}\text { Valori di } \\
\text { riferimento } \\
\text { italiani }\end{array}$ \\
\hline Trippoli S. et al. & Italia & NSCLC & $\begin{array}{l}\text { Correlazione tra } \\
\text { Qol e Utilità }\end{array}$ & $\begin{array}{l}95 \\
\text { Nessun rifiuto }\end{array}$ & self, in clinica & $\begin{array}{l}\text { Valori di } \\
\text { riferimento } \\
\text { italiani }\end{array}$ \\
\hline
\end{tabular}

Tabella 6

Uso del MOS SF-36 
sultata essere 36,8 (DS 9,8), quella delle dimensioni “mentali" 43,0 (DS 11,3). Il punteggio medio ottenuto all'EuroQOL è stato 0,58 (DS 0,32) nella versione autoclassificativa (SC) e 0,58 (DS $0,20)$ nella versione analogica visiva (VAS). Tra le variabili cliniche che influenzano la qualità di vita e l'utilità, la presenza di metastasi ha avuto il maggior impatto. Le misure EuroQOL ottenute da questi pazienti potranno essere utili per la valutazione dei rapporti di costo-utilità delle terapie dei NSCLC.

Sono attualmente in corso tentativi di convertire i valori ottenuti con il MOS SF-36 in una misura di utilità.

Gli studi di Dahloef et al (18) e di Pariente et al (30) hanno dimostrato gli effetti positivi di terapie, nuove e correnti fornendo ai decisori sanitari informazioni che possono essere importanti per la scelta dei trattamenti adeguati. O'Conor et al hanno riscontrato una rilevanza significativa di 6 delle 8 dimensioni del MOS SF-36 nei confronti della gravità dei sintomi. In questo studio è stata inoltre utilizzata una metodica, nota con il nome di metodo della valutazione contingente (CVM), per misurare la disponibilità a pagare (willingness to pay WTP), intesa come misura del beneficio individuale. I risultati, che indicano che in media (mediana) i pazienti sono disposti a pagare
27,24 USD (87,74 USD) al mese per ridurre del $25 \%$ gli episodi di nicturia e di incontinenza e 75,92 USD (244,54 USD) al mese per ridurli del $50 \%$, possono essere direttamente comparati al costo delle terapie. C'è però da osservare, a mo' di nota, che i risultati in termini di WTP forniti da questo studio sono da prendere con cautela, in quanto il campione analizzato non era randomizzato e una quota significativa dei pazienti è stata esclusa perché considerava troppo complicata la compilazione del questionario.

Tre delle sei ricerche che hanno usato l'EQ5D (Tabella 7) hanno valutato la costo-efficacia o la costo-utilità dei trattamenti studiati. I miglioramenti/peggioramenti della qualità di vita si sono rivelati componenti importanti per la valutazione degli esiti e dei costi di una terapia.

Uyl-de Groot et al (37) hanno riscontrato che la somministrazione di GM-CSF a pazienti con neutropenia febbrile non risulta in una riduzione significativa della durata dell'ospedalizzazione, nonostante un ripristino più rapido della conta leucocitaria, né migliora la qualità di vita dei pazienti. In base a queste osservazioni, gli autori concludono che

\begin{tabular}{|c|c|c|c|c|c|c|}
\hline Lavoro & Luogo & Area clinica & Obiettivo & $\begin{array}{l}\text { Dimensione } \\
\text { del campione }\end{array}$ & Somministrazione & $\begin{array}{l}\text { Gruppo di } \\
\text { controllo }\end{array}$ \\
\hline $\begin{array}{l}\text { Uyl-de Groot } \\
\text { CA.et al. }\end{array}$ & Olanda & $\begin{array}{l}\text { Neutropenia } \\
\text { febbrile correlata } \\
\text { a terapia } \\
\text { antineoplastica }\end{array}$ & $\begin{array}{l}\text { Esaminare la } \\
\text { Qol con GM- } \\
\text { CSF }\end{array}$ & $\begin{array}{l}134 \\
90(67 \%) \\
\text { complete }\end{array}$ & self, in clinica & Placebo \\
\hline Gudex CM. et al. & GB & Distonia & $\begin{array}{l}\text { Misurare il } \\
\text { Beneficio della } \\
\text { tossina botulinica }\end{array}$ & $\begin{array}{l}196 \\
130(66 \%) \\
\text { complete }\end{array}$ & $\begin{array}{l}\text { Intervista }+ \text { follow- } \\
\text { up per posta }\end{array}$ & $\begin{array}{l}\text { Popolazione } \\
\text { generale GB } \\
\text { (VAS e TTO) }\end{array}$ \\
\hline Johnson JA. et al. & USA & & $\begin{array}{l}\text { Valutazione di } \\
\text { EQ-5D in un } \\
\text { campione di } \\
\text { adulti USA }\end{array}$ & $\begin{array}{l}3500 \\
905(25.8 \%) \\
\text { utilizzabili }\end{array}$ & self, per posta & \\
\hline Badia X. et al. & Spagna & $\begin{array}{l}\text { Patologia } \\
\text { cronica }\end{array}$ & $\begin{array}{l}\text { Influenza } \\
\text { dell'esperienza di } \\
\text { malattia }\end{array}$ & $\begin{array}{l}480 \\
\text { 120: malati, 360: } \\
\text { sani }\end{array}$ & $\begin{array}{l}\text { da } 2 \text { studi } \\
\text { precedenti }\end{array}$ & Soggetti sani \\
\hline Griffin AD. et al. & $\begin{array}{l}\text { GB, Nord } \\
\text { America, } \\
\text { Europa, Israele }\end{array}$ & Influenza A e B & $\begin{array}{l}\text { Valutare la costo } \\
\text { efficacia di } \\
\text { Zanamivir in } \\
\text { pazienti ad alto } \\
\text { rischio }\end{array}$ & $\begin{array}{l}21 \text { pazienti e } 8 \\
\text { MMG britannici }\end{array}$ & Da 2 fonti diverse & $\begin{array}{l}\text { Valori basali } \\
\text { ottenuti per } \\
\text { ciascun paziente }\end{array}$ \\
\hline Trippoli S. et al. & Italia & NSCLC & Qol e Utilità & $\begin{array}{l}95 \\
\text { Nessun rifiuto }\end{array}$ & self, in clinica & $\begin{array}{l}\text { Valori di } \\
\text { riferimento GB }\end{array}$ \\
\hline
\end{tabular}

GM-CSF: Granulocyte-macrophage colony-stimulating factor

NSCLC: Non-Small Cell Lung Cancer

MMG: Medico di Medicina Generale

Tabella 7 Uso di $E Q-5 D$ 
l'intervento studiato non può essere considerato costo-efficace.

Griffin et al (21) riferiscono di aver stimato che l'influenza riduce l'utilità di 0,083 per giorno $(n=21)$. Il valore basale era uguale a 0,817 , il punteggio dello stato di salute influenza = 0,066. È risultato un costo di $3900 £$ per QALY, spese di degenza escluse ( $7490 £$ se si includono tali spese). Una curva di accettabilità ha dimostrato con una confidenza del $90 \%$ che il zanamivir risulterebbe costo-efficace a un livello di $8000 £$ per QALY. In base a ciò si potrebbero ottenere significativi benefici sanitari trattando pazienti ad alto rischio con zanamivir. Secondo gli autori il costo per QALY di questi pazienti è comparabile a quello di altre terapie comunemente impiegate.

Nello studio di fattibilità condotto da Gudex et al, l' analisi di costo-utilità ha confrontato la terapia con la tossina botulinica (BT) nei pazienti con distonie con due serie distinte di valori normali di popolazione. Le stime di costo per QALY variavano considerevolmente a seconda del tipo di distonia, della durata del trattamento con BT, del tipo di valori di preferenza per gli stati di salute utilizzato e delle caratteristiche basali dei pazienti studiati. Le stime di costo per QALY ottenute usando i valori di preferenza VAS e TTO della popolazione britannica sono state di 30.000-50.000£ e di 45.000-55.000£, rispettivamente. La natura della patologia e il suo trattamento ciclico hanno comportato difficoltà pratiche nel reclutamento dei pazienti, nella somministrazione dei questionari e nella stima dei QALY guadagnati. Gli autori suggeriscono che la formula da loro sviluppata per il calcolo del guadagno in QALY potrebbe essere applicata in altre analisi di costo-utilità che esaminino un disturbo cronico ma variabile nel tempo.

Le valutazioni degli stati di salute compresi nell'EQ-5D sono state ottenute in svariati studi di popolazione, usando campioni provenienti, ad esempio, dall'Olanda, dalla Svezia, dalla Norvegia e dalla Gran Bretagna. Sebbene l'inglese sia stata la lingua adottata per costruire il modello comune dello strumento di misura, sono state sviluppate e validate traduzioni in tutte le lingue parlate negli Stati che facevano parte del gruppo EuroQOL originale.

Da un punto di vista politicoprogrammatico, se uno strumento come l'EQ$5 \mathrm{D}$ dovesse venire utilizzato come base di decisioni allocative sarebbe preferibile utilizzare un sistema di ponderazione derivato dai valori di preferenza delle popolazioni dei singoli Paesi, in quanto maggiormente rappresentativo. Per tale motivo Johnson et al (27) si sono pro- posti di derivare una sistema di ponderazione basato sulla popolazione statunitense per la serie di stati di salute considerati nel questionario EQ-5D. Sono state calcolate le medie e le mediane delle valutazioni espresse per 45 stati di salute, morte e incoscienza incluse. Successivamente è stato sviluppato un sistema ponderativo basato su una regressione lineare ordinaria per attribuire un punteggio agli stati di salute non direttamente considerati. Le valutazioni ottenute per gli stati di salute EQ5D standard hanno mostrato caratteristiche simili a quelle espresse attraverso le VAS in precedenti studi condotti in Europa. Il modello a regressione lineare si è adattato relativamente bene ai dati osservati, raggiungendo un $\mathrm{R} 2$ aggiustato di 0,42. Le valutazioni EQ-5D fornite da adulti statunitensi hanno mostrato un comportamento simile a quello osservato in studi di valutazione precedenti. I test diagnostici applicati al modello iniziale, tuttavia, hanno dimostrato che esso conteneva degli errori di specificazione, per cui la possibilità di generalizzare i risultati all'intera popolazione americana adulta potrebbe essere inficiata dalle violazioni delle assunzioni del modello di regressione lineare.

L'articolo di Badia et al (14) ha comparato le valutazioni espresse per alcuni stati di salute da pazienti affetti da patologie croniche con le valutazioni ottenute da soggetti relativamente sani. Queste misure sono state ottenute con l'EQ-5D e hanno mostrato che le valutazini differiscono in maniera significativa, in particolare per gli stati di salute peggiori. I soggetti sani hanno assegnato valori negativi ad alcuni stati di salute ipotetici, rivelando di considerarli peggiori della morte, mentre i malati cronici hanno attribuito punteggi positivi a tutti gli stati di salute. Da questi risultati nascono domande difficili, riguardanti la scelta dei soggetti le cui valutazioni sono da considerare per l'applicazione delle misure dello stato di salute ad analisi cliniche ed economiche, particolarmente quelle condotte per decidere l'allocazione di risorse limitate.

Alcune questioni, come il tasso di risposta e le motivazioni dei non rispondenti, vengono spesso affrontate in questi articoli. Viene riferito che la valutazione della morte all'interno dell'EQ-5D ha causato tensione e confusione $(1,27)$, particolarmente nei pazienti. Inoltre, nessuno di questi articoli ha tentato di calcolare i costi intangibili, benché essi possano essere considerati nelle analisi di costo della malattia.

È molto probabile che le informazioni riguardanti la valutazione della qualità di vita correlata alla salute diventeranno sempre più numero- 
se. Siamo fiduciosi nei progressi nello sviluppo delle metodologie e nell'affinamento degli strumenti di misura, che dovranno riflettere il vero valore dell'impatto delle patologie e dei trattamenti sui pazienti e sulla società nel suo insieme al fine da fornire una base più accurata per i processi decisionali.
Questo articolo è frutto del lavoro svolto dall'Autrice durante lo stage compiuto presso Adis International Italy, con la supervisione di Carlo Lucioni

\section{BIBLIOGRAFIA}

1. Selai C. and Rosser R. Eliciting EuroQol Descriptive Data and Utility Scale Values from Inpatients. PharmacoEconomics 8(2): 147-158, 1995

2. Lucioni C. Quality of life in the economic evaluation. Progress in Clinical Pharmacy, Clinical Trials and Pharmacoepidemiology, edited by G. Scroccaro, N. Martini, J.P. Delporte, et al. European Society of Clinical Pharmacy, 1994

3. Coons SJ, Rao S, Keininger DL, et al. A comparative review of generic quality-of-life instruments. PharmacoEconomics 17(1) $13-35,2000$

4. Ware JE, Kosinski M, Keller SD. SF-36 physical and mental health summary scales: a user's manual. The Health Institute, New England Medical Center. Boston Massachusetts, Dec. 1994. 4th printing.

5. Brazier J, Usherwood T, Harper R, et al. Deriving a preference-based single index from the UK SF-36 health survey. J Clin Epidemiol Vol. 51, No. 11,pp. 1115-1128,1998

6. Arpinelli F, Visonà G, Bruno R, et al. Health-related quality of life in asymptomatic patients with HIV: evaluation of the SF-36 health survey in Italian patients. Pharmacoeconomics 18(1): 63-72, 2000

7. Pibernik-Okanovi M, Szabo S, Metelko Z. Quality of life following a change in therapy for diabetes mellitus. PharmacoEconomics 14(2): 201-207, 1998

8. Drummond MF, O'Brien B, Stoddart GL, et al. Methods for the evaluation of health care programmes. 2 nd ed. Oxford: Oxford University Press, 1997

9. Green C, Brazier J, Deverill M. Valuing health-related quality of life: a review of health state valuation techniques. PharmacoEconomics 17 (2): 151-165, 2000

10. Voruganti LNP, Awad AG, Oyewumi LK, et al. Assessing health utilities in schizophrenia: a feasibility study. PharmacoEconomics 17(3): 273-286, 2000

11. EQ-5D user guide. A measure of Health-related quality of life developed by the EuroQOL group. 5th issue, April. 1998

12. Ackerman SJ, Sullivan EM, Beusterien KM, et al. Cost effectiveness of recombinant human insulin-like growth factor I therapy in patients with ALS. PharmacoEconomics 15(2): 179-195, 1999

13. Aoki FY, Fleming DM, Griffin AD, et al. Impact of zanamivir treatment on productivity, health status and healthcare resource use in patients with influenza. PharmacoEconomics 17(2): 187-195, 2000

14. Badia X, Herdman M, and Kind P. The influence of ill-health experience on the valuation of health. Pharmacoeconomics 13 (6): 687-696, 1998

15. Boyer P, Danion JM, Bisserbe JC, et al. Clinical and economic comparison of sertraline and fluoxetine in the treatment of depression. PharmacoEconomics 13 (1-1): 157-169, 1998

16. Cremieux P-Y, Finkelstein SN, Berndt ER, et al. Cost effectiveness, quality-adjusted life-years and supportive care: recombinant human erythropoietin as a treatment of cancer-associated anaemia. Pharmacoeconomics $16(5 \mathrm{Pt} 1)$ : 459-472, 1999

17. Coyle D, Cranney A, Lee KM, Cost effectiveness of nasal calcitonin in postmenopausal women: use of Cochrane Collaboration methods for meta-analysis within economic evaluation. Pharmacoeconomics 19(5 Pt 2):565-75, 2001

18. Dahlöf C, Bouchard J, Cortelli P, et al. A multinational investigation of the impact of subcutaneous sumatriptan. II: health-related quality of life. 11 Pharmacoeconomics (Suppl. 1): 24-34, 1997

19. Evans KW, Boan JA, Evans JL, et al. Economic evaluation of oral sumatriptan compared with oral caffeine/ ergotamine for migraine. Pharmacoeconomics 12 (5): 565-577, 1997

20. Fragnani F, Bouvenot G, Valat J-P, et al. Medico-economic analysis of diacerein with or without standard therapy in the treatment of osteoarthritis. Pharmacoeconomics 13 (1-1): 135-146, 1998 
21. Griffin AD, Perry AS, Fleming DM. Cost-effectiveness analysis of inhaled zanamivir in the treatment of influenza A and B in high-risk patients. Pharmacoeconomics 19(3): 293-301, 2001

22. Griffiths RI, Bleecker GC, Jabs DA, et al. Pharmacoeconomic analysis of 3 treatment strategies for cytomegalovirus retinitis in patients with AIDS. Pharmacoeconomics 13(4): 461-474, 1998

23. Gudex CM, Hawthorne MR, Butler AG, et al. Measuring patient benefit from botulinum toxin in the treatment of dystonia: feasibility of cost-utility analysis. Pharmacoeconomics 12 (6): 675-684, 1997

24. Hakkaart-van Roijen L, Beckers A, Stevenaert A, et al. The burden of illness of hypopituitary adults with growth hormone deficiency. Pharmacoeconomics 14(4): 395-403, 1998

25. Hatoum HT, Kong SX, Kania CM, et al. Insomnia, health-related quality of life and healthcare resource consumption: a study of managed-care organisation enrollees. Pharmacoeconomics 14(6): 629-637, 1998

26. Hummel S, Piercy J, Wright R, et al. An economic analysis of the Survival and Ventricular Enlargement (SAVE) study: application to the United Kingdom. Pharmacoeconomics 12 (2 Pt 1): 182-192, 1997

27. Johnson JA, Coons SJ, Ergo A, et al. Valuation of EuroQOL (EQ-5D) health states in an adult US sample. Pharmacoeconomics 13(4): 421-433, 1998

28. Mittmann N, Trakas K, Risebrough N, et al. Utility scores for chronic conditions in a community-dwelling population. Pharmacoeconomics 15(4): 369-376, 1999

29. O'Conor RM, Johannesson M, Hass SL, et al. Urge incontinence: quality of life and patients' valuation of symptom reduction. Pharmacoeconomics 14(5): 531-539, 1998

30. Pariente PD, LePen C, Los F, et al. Qualilty-of-life outcomes and the use of antihistamines in a French national population-based sample of patients with perennial rhinitis. Pharmacoeconomics 12(5): 585-595, 1997

31. Rosner AJ, Grima DT, Torrance GW, et al. Cost effectiveness of multi-therapy treatment strategies in the prevention of vertebral fractures in postmenopausal women with osteoporosis. Pharmacoeconomics 14(5): 559-573, 1998

32. Shimbo T, Hira K, Takemura M, et al. Cost-effectiveness analysis of dopamine agonists in the treatment of Parkinson's disease in Japan. Pharmacoeconomics 19(8): 875-886, 2001

33. Smith KJ, Roberts MS. Cost effectiveness of early treatment with oral aciclovir in adult chickenpox. Pharmacoeconomics 13(5-5): 645-651, 1998

34. Torrance G, Walker V, Grossman R, et al. Economic evaluation of ciprofloxacin compared with usual antibacterial care for the treatment of acute exacerbations of chronic bronchitis in patients followed for 1 year. Pharmacoeconomics 16 (5 Pt 1): 499-520, 1999

35. Trallori G, Messori A. Drug treatments for maintaining remission in Crohn's disease: a life time cost-utililty analysis. Pharmacoeconomics 11(5): 444-453, 1997

36. Trippoli S, Vaiani M, Lucioni C, et al. Quality of life and utility in patients with non-small cell lung cancer. Pharmacoeconomics 19(8): 855-863, 2001

37. Uyl-de Groot CA, Vellenga E, de Vries EGE, et al. Treatment costs and quality of life with granulocyte-macrophage colony-stimulating factor in patients with antineoplastic therapy-related febrile neutropenia: results of a randomised placebo-controlled trial. Pharmacoeconomics 12(3): 351-360, 1997 